\title{
Criminologie
}

\section{Approche institutionnelle et impact de la sortie de psychotique dangereux}

\section{Bruno Gravier et André Surprenant}

Volume 15, numéro 2, 1982

Criminels et psychiatrie

URI : https://id.erudit.org/iderudit/017161ar

DOI : https://doi.org/10.7202/017161ar

Aller au sommaire du numéro

Éditeur(s)

Les Presses de l'Université de Montréal

ISSN

0316-0041 (imprimé)

1492-1367 (numérique)

Découvrir la revue

Citer cet article

Gravier, B. \& Surprenant, A. (1982). Approche institutionnelle et impact de la sortie de psychotique dangereux. Criminologie, 15(2), 83-92.

https://doi.org/10.7202/017161ar d'utilisation que vous pouvez consulter en ligne.

https://apropos.erudit.org/fr/usagers/politique-dutilisation/ 
APPROCHE INSTITUTIONNELLE ET IMPACT DE LA SORTIE DU PSYCHOTIQUE DANGEREUX Bruno Gravier, M.D.* André Surprenant *

Le texte que nous présentons est issu de notre pratique au sein d'une unité de traitement pour psychotiques dangereux à l'Institut Philippe Pinel. Malgré l'abondante littérature et la profusion d'expériences ayant trait à la pratique institutionnelle, une réflexion sur les approches psychothérapiques dans un univers clos, comme l'est celui de l'I.P.P.M., est toujours malaisée, car comme le souligne Koechlin (8) :

1. le cadre est l'institution dont la complexité et la variabilité sont évidemment plus grandes que le cadre des autres psychothérapies; la pregnance des contraintes hospitalières risque de détruire ce que l'on avait pu estimer être le cadre nécessaire, elle risque également d'infléchir continuellement les règles et protocoles qu'on aurait pu définir;

2. la relation thérapeutique s'opère entre le malade et le thérapeute mais aussi entre le malade et le personnel soignant, les autres malades et même l'institution;

3 . enfin, les médiateurs de cette relation sont certes verbaux, mais plus encore matériels et partie constituante de l'institution.

Le caractère coercitif de l'Intitut Pinel, rendu nécessaire par la spécificité de la population qui y séjourne, ajoute une donnée supplémentaire qui ne facilite pas l'élaboration d'une politique orientée vers le traitement et la réinsertion sociale.

Les rites asilaires sont inhérents à toute institution totalitaire (Goffman, 4), et l'I.P.P.M. ne déroge pas à la règle.

Le caractère marginal de l'Institut à mi-chemin entre l'hôpital psychiatrique et la prison est source d'une profonde ambivalence qui rejaillit aussi bien sur nous tous qui y travaillons que sur les patients qui y vivent; ce caractère marginal que l'on ne retrouve que dans peu d'institutions à travers le monde fait que le système de référence et de communication qui permet d'avoir une réflexion et développer une approche est très restreint, ce qui nous oblige à tâtonner longuement avant de pouvoir réellement nous identifier à un type de pratique.

* Bruno Gravier, docteur, Institut Philippe Pinel.

* André Surprenant, M.A. psychologue, Institut Philippe Pinel. 
Spécificité de la clientèle de l'institut

Cette ambivalence de l'Institut se concrétise dès que l'on essaye d'identifier qui l'on soigne ou plutôt qui l'on traite. En effet, si les souffrances du schizophrène morcellé ne nous laissent pas indifférents et nous amènent à une certaine empathie naturelle, la dimension criminalisée de leur vécu provoque un recul et une méfiance dont il nous est difficile de nous départir. Nous sommes avant tout une présence de sociopathes, terme qui recouvre une série de situations jusque-là arbitrairement séparées (Hochmann,5). L'étrangeté du fou renforce la déviance du criminel et élargit l'espace entre la société normalisée et honnête, nous, et les fous dangereux, eux. On comprend aisément qu'il n'est pas facile d'abolir ou de restreindre un tel espace, pourtant nécessaire si l'on veut établir le contact indispensable à toute action thérapeutique.

Notre postulat a été tout au long de notre pratique de nous adresser à la personne souffrante plutôt qu'au déviant exclu, ce qui ne nous permet cependant pas de faire l'économie de la réflexion sur la dangerosité potentielle que représentent ceux à qui nous nous adressons. Cette dangerosité prend d'ailleurs un aspect obsédant dans tous nos rapports avec les patients.

De toute façon, cette dangerosité ne peut être exclue, car nos patients ont déjà effectué un ou plusieurs passages à l'acte, souvent de façon dramatique, passages à l'acte qui constituent leur billet d'entrée dans ce lieu qui représente dans l'imaginaire social, un enfer d'où on ne devrait pas sortir. Le mandat de garde pour une durée indéfinie, étiquette collée sur le dos de la majorité de nos patients "acquittés " pour aliénation mentale, illustre bien cette marque d'infamie que la société confère à ceux qu'ils l'ont troublée de façon peu commune.

Peu commun est bien le qualificatif qui peut s'appliquer à nos patients, car s'il est relativement aisé de poser un diagnostic de psychose devant des manifestations délirantes et d'adopter une attitude thérapeutique en rapport, la bizarrerie et l'incompréhensibilité de beaucoup d'actes homicidaires ne cessent pas de nous questionner.

Aussi, c'est la façon dont s'articule le délit avec la personnalité du patient, son vécu antérieur et son vécu actuel qui va nous permettre d'aborder cette problématique, c'est dire ici la nécessité d'une approche thérapeutique fortement individualisée. 


\section{But du traitement}

La principale fonction d'un établissement de soins psychiatriques est de fournir aux patients une intervention à caractère restaurateur, et ce malgré la lourde hypothèque qui pèse sur eux. En effet, il est important, à travers les relations qu'il va connaître à l'Institut, que le patient vive une expérience que son passé, son histoire et son organisation psychique ne lui ont pas permis de vivre et d'intégrer (Racamier, 12). L'hôpital ne peut être qu'un lieu qui prépare à un ailleurs où le patient sera plus confronté à la réalité, à sa réalité. Le séjour à l'Institut comme celui de tout autre hôpital, ne peut et ne doit être considéré que comme une étape, et non comme une fin en soit. Cette exigence, nos patients la portent en eux, et la verbalisent de bien des façons qui nous apparaissent la plupart du temps intempestives. Pour le psychotique, cette exigence devient une question de vie ou de mort, et bien souvent une modalité relationnelle à travers laquelle il testera ses thérapeutes ainsi que toute l'équipe soignante, construisant et détruisant les liens fragiles existants entre lui et eux. Tout en véhiculant son angoisse à travers son interrogation répétitive, le patient demande une date ou une échéance comme pour fixer un terme à un conflit insoluble entre la tristesse et la monotonie de sa vie actuelle qu'il attribue aux murs de l'institution et l'impossibilité déjà maintes fois expérimentée de sa vie au-dehors.

Cette quête incessante traduit bien la tendance profonde du psychotique à chercher son identité ou plutôt son identification. Cette recherche s'actualise le plus souvent dans de nombreuses identifications primitives et fusionnelles, qui le fascinent et l'aliènent encore plus (Racamier, 13). Ces tendances se concrétisent petit à petit par la naissance d'un sentiment d'appartenance au statut de détenu, statut qu'il adopte d'autant plus facilement qu'il côtoie continuellement d'authentiques délinquants. Ce sentiment d'appartenance lui permet d'estimer la durée nécessaire à sa réhabilitation en comparant son temps de séjour avec les normes qu'il pense être en vigueur dans l'Institut. Ainsi, magiquement, en acquittant sa dette envers la société, il sera délivré du fardeau que constitue le crime qu'il a commis et qu'il n'a jamais compris et sera par là même guéri. Ce processus lui permet une fois de plus de dénier le réel et d'éviter ainsi une nouvelle fois d'avoir à supporter l'anglisse d'anéantissement (Escande, 3). Bien évidemment, cet espoir est de nouveau déçu et les représentants de l'institution se font les réceptacles des mauvais objets projetés par les patients. 
Un de nos patients illustre bien ce type de comportement.

Âgé de trente-huit ans, il a connu ses premiers épisodes psychotiques dès l'âge de 20 ans; gros travailleur, il a été employé comme assistant-plombier pendant de longues années. Par la suite, son existence a suivi le cours chaotique que la schizophrénie imprime à de nombreux patients, jusqu'au soir où délirant et halluciné, se croyant au cœur de la guerre mondiale, il tue à coups de couteau un de ses voisins, infirme et assisté social, dont il s'occupait occasionnellement. Il se trouve depuis trois ans à l'Institut, de plus en plus apragmatique, périodiquement halluciné et délirant malgré une forte chimiothérapie.

Pendant la plupart des rencontres que nous avons avec lui, que ce soit individuellement ou en groupe, il monopolise la période pendant de longs moments pour exprimer son ressentiment envers l'Institut qui le garde de façon injuste et disproportionnée avec l'acte qu'il a commis - il n'a tué qu'un quasi mendiant - et qui ne favorise pas la réhabilitation de ses pensionnaires, alors que lui se dit prêt à retourner au travail. Petit à petit, il est apparu que son attitude témoignait plutôt d'un refus de parler de certains aspects de son passé et d'aborder de ce fait des problématiques angoissantes.

Ainsi, de cette façon les psychotiques éludent la démarche qui leur permettrait de retrouver une cohérence interne, et se contentent d'un simple "plaquage du sur-moi " (Koehlin, 8), plaquage renforcé par les exigences institutionnelles qui demandent, avant que quelques procédures de sortie ne soient engagées, que le malade "fonctionne", c'est-à-dire que son comportement témoigne de sa réadaptation. C'est encore bien souvent une porte de sortie dans laquelle les patients s'engouffrent pour ne pas avoir à aborder leurs conflits. À cet égard, nous pouvons rapporter une anecdote significative.

Un patient est suivi régulièrement en clinique externe depuis sa sortie. Au cours de ses entrevues, il nous avoue avoir souffert pendant une grande partie de son hospitalisation de phobies d'impulsion homicidaire à chaque fois qu'il se rendait à une activité. Il nous fait part en même temps de l'angoisse que suscitait chez lui de telles phobies, et de ses efforts pour les dissimuler aux yeux du personnel de peur que celui-ci perde la bonne image que le patient avait essayé de véhiculer.

Comme on le voit, les résistances des patients à nous communiquer leur vécu vont se loger dans bien des recoins de la vie institu- 
tionnelle. Mais ces mécanismes de défense au moyen desquels le psychotique pourra nier sa pathologie et se présenter à nos yeux comme un sociopathe réadapté sont bien fragiles et suffisent rarement à contenir les pulsions destructrices et autodestructrices. En effet, il n'est pas rare qu'alors que tout va bien et qu'un patient semble prêt à quitter l'institution qu'il "décompense " brutalement, sans crier gare.

Ce terme de décompensation, vague et fourre-tout, recouvre bien des aspects que prend la plainte du psychotique qui ne se sent pas prêt à être abandonné par une institution qui, malgré ses carences, constitue le dernier rempart contre sa psychose. Parfois, cette plainte prend la forme d'un nouveau processus délirant aigu qui nous renverra à notre image dévalorisée de mauvais thérapeute. Le plus souvent, c'est sous la forme de comportements déviants par rapport au mode de fonctionnement de l'unité que celle-ci se présente : refus de participation, retrait de la vie collective, etc. C'est dire l'importance pour nous de "pénétrer par l'identification, le vécu étrange et angoissé des psychotiques et ensuite de revenir de cette identification pour éviter ainsi ce que l'on peut appeler l'identification " captive " (Racamier, 12). En effet, pour nous, l'aspect fondamental du traitement est de permettre à nos patients de pouvoir effectuer le travail de réparation tel que l'ont décrit les auteurs kleiniens. Ce travail ne peut s'effectuer qu'en favorisant le désir et la capacité de reconstituer le bon objet interne et externe. Les restaurations réussies des objets du psychotique lui permettent d'augmenter sa confiance en sa capacité d'aimer et ainsi sa propre haine devient moins effrayante. Cette étape indispensable passe obligatoirement par la répétition de pertes et de récupérations ressenties comme une destruction par la haine et une re-création par l'amour (Segal, 17). Seule une relation transférentielle authentique peut permettre aux patients de franchir ces étapes décisives, il est donc fondamental pour nous de pouvoir comprendre leurs résistances.

Difficultés de la communication et impact de la violence du patient sur les réactions contre-transférentielles

Plusieurs auteurs ont récemment insisté sur les réactions et les attitudes que pouvaient susciter dans une équipe soignante d'un service hospitalier la présence de patients potentiellement violents. Ces réactions sont variées et visent le plus souvent à se protéger d'affects angoissants et insécurisants (Rosen, 15). L'attitude la plus fréquemment mentionnée est le déni de cette violence potentielle (Cornfield 
(1), Rosen (15), Hay (5) ). Ce déni entraîne un détachement émotionnel apparent, ou un investissement plus grand dans d'autres patients moins menaçants et eux-mêmes menacés, ou même un déplacement et une diffusion de l'hostilité envers les structures hospitalières (Cornfield, 1). Lion et Pasternak ont aussi souligné la fascination que pouvait exercer chez certains membres du personnel le récit des exactions de certains sujets violents. Tous ces mécanismes visent en fait à éviter d'aborder en face le problème que peut nous poser la perception de la violence des patients, à plus forte raison s'ils sont psychotiques, car ceux-ci ont de plus pour objectif de nous rendre fou, en attaquant ce qui nous permet la liaison, la mise en signification et l'organisation de l'expérience (Hochmann 7, Searles, 16). Lion et Coll soulignent que ces réactions de l'équipe envers un patient constituent un renforcement important de son aliénation et peuvent éventuellement aggraver sa psychose. De fait, de telles attitudes que nous avons bien souvent constatées entraînent de considérables distorsions dans les communications interpersonnelles au sein des unités (Cornfield, 1).

On peut imaginer facilement le désarroi des patients qui, comme l'a montré Virkkunen (18), se trouvent souvent pendant la période précédant leur délit dans une détresse relationnelle importante, et qui se retrouvent brutalement plongés dans un environnement inhabituel caractérisé par une incessante activité, la profusion de stimuli et une organisation sociale relativement peu structurée (Rosen 15, Rhine 14, Weisman 19) ou du moins structurée d'une façon inhabituelle pour eux. Ce désarroi ne peut qu'être renforcé par les réactions contre-transférentielles de l'équipe et entraîne chez le patient inévitablement une impossibilité à s'investir dans des tâches thérapeutiques (Rosen 15). On se retrouve embarqué dans une spirale ascendante où l'angoisse des uns renforce la maladie des autres. Si l'on ne peut pas déchiffrer les messages et les demandes d'aide qui circulent parfois même par le biais des faits anodins (dispense d'activité, demande de changement de chambre), une atmosphère de tension et de peur finit par s'installer de façon insurmontable. Et la seule façon de résoudre une telle situation est bien souvent le passage à l'acte, ce passage à l'acte comme toute autre manifestation du vécu du patient doit être compris et d'une certaine façon accepté, car comme le souligne Koechlin (8) : «l'acceptation par l'institution de "l'acting out " entraînera bien souvent non pas un renforcement des réactions de défense vis-à-vis d'elle, mais au contraíre un renforcement de la relation. " 
Un malade de l'unité a pour habitude à chacune de ses poussées délirantes et angoissées de se retirer isolément dans sa chambre et de jeter tous ses effets personnels par la fenêtre. Pendant longtemps la réponse de l'équipe fut de le punir et de l'isoler pour de tels comportements. Par la suite, nous avons compris que le patient par son attitude nous signifiait qu'il avait conscience de sa violence et de ses pulsions agressives incontrôlables et qu'il préférait les adresser à des objets plutôt que de nous agresser physiquement. Petit à petit, chacun de ses acting out fut l'occasion de renouer des liens avec lui en l'accompagnant au-dehors chercher ses effets et en le laissant simplement se reposer à sa chambre. Nous avons pu constater dès lors que ses agirs violents se rarefiaient pour ne plus se manifester que de façon sporadique. Parallèlement, son implication dans les séances de psychothérapie s'améliorait ainsi que la qualité de son transfert.

Tout ceci illustre bien l'extrême sensibilité du psychotique à tout ce qui l'entoure et principalement à l'insécurité qui peut régner. Aussi, nous avons pu constater que plus nos interventions étaient rassurantes, et que les intervenants s'exprimaient sur leur vécu face aux malades dont ils avaient la charge, plus la relation avec ceux-ci s'améliorait et plus facilement nous pouvions mettre à jour les conflits profonds de nos patients et ainsi apprécier le potentiel de danger que ceux-ci représentent face à nous.

Evaluation du potentiel de dangerosité et l'impact d'une politique de sorties

Il apparaît évident que le thérapeute doit être à l'écoute de ses propres peurs et de son hostilité. Il lui faut aussi se méfier de l'effet de ces sentiments, suscités par l'impact des projections du patient, dans l'appréciation de la dangerosité de celui-ci (Lion, 9). Quand cette appréciation est l'affaire d'une personne, elle est intimement liée à la subjectivité inhérente à toute relation, et la vision qui en découle ne peut être que parcellaire. En fait, la détermination de la conduite à tenir avec chaque patient ne peut être que le fruit d'une confrontation bien souvent passionnée car les vécus de chaque intervenant face au patient sont souvent bien différents et les attitudes qui en découlent sont parfois diamétralement opposées. Là encore, il faut se garder de faire le jeu de la psychose qui projette ses tendances morcellantes et irréconciliables sur l'équipe. Il faut pouvoir provoquer un échange dialectique plutôt qu'un affrontement 
sans fin, ce qui a souvent entraîné malheureusement l'éclatement des équipes. La dimension collective de la réflexion autour d'un patient nous paraît être la seule possibilité d'arriver à une vision plus objective des possibilités et des demandes du patient, "car elle répond à de réelles nécessités économiques et dynamiques en autant que l'on considère les facteurs d'investissement transférentiel » (Racamier, 11).

La décision de faire bénéficier un patient d'une sortie même temporaire n'est jamais une décision anodine, bien au contraire, c'est souvent à cette occasion que resurgissent des vieux démons exorcisés par l'enfermement du patient dangereux. Dès le moment où l'hypothèse d'une sortie est avancée, l'agir, les différents comportements, le discours et la capacité d'autocritique du patient sont soigneusement décortiqués et critiqués, et bien souvent l'analyse de données purement factuelles devient une résistance de plus, occultant le cheminement du patient et le nôtre face à lui, et marquant l'angoisse que produit chez nous la perspective de la mise en liberté prochaine d'un individu potentiellement dangereux, ou du moins qui l'a été à une période de sa vie. Là encore, l'approche collective peut avoir un caractère de diffusion désangoissante (Racamier, 11). Bien souvent, cette prise de décision entérinée par les instances institutionnelles entraîne chez le personnel un désinvestissement des relations et des liens noués avec le patient, comme si elle correspondait à la fin du mandat social donné à l'équipe. Pourtant, c'est la période cruciale pour les patients, c'est le moment où ils sont confrontés avec l'épreuve de réalité, et c'est le moment que choisit l'institution pour devenir une mère délaissante. Nous avons pu constater à maintes reprises la valeur cathartique et bénéfique pour le traitement d'une sortie même isolée. Si la relation transférentielle est solide, elle pourra permettre au patient de communiquer son vécu soudainement enrichi par la redécouverte de l'extérieur et ainsi l'aider à se différencier, permettant de cette façon "l'heureux aboutissement de ce processus maturant dit de "personnation » qui délimite le dépassement de la zone de fonctionnement psychotique et l'entrée dans la problématique névrotique ou normale " (Dubor, 2 ).

Il nous apparaît important de dissocier dans la problématique de la confrontation du patient avec l'extérieur, les sorties temporaires, le plus souvent accompagnées et la sortie définitive si angoissante. Car la sortie temporaire permet toujours au patient de sortir de cette période de gestation et de dépression si désespérante pour toute l'équipe et pendant laquelle se développent insidieusement les 
germes de la chronicisation. De plus, ces sorties, lorsqu'elles surviennent dans une perspective de sortie définitive à court terme ne font que renforcer l'anxiété du patient qui se sent guetté et épié dans ses réactions, car il se rend bien compte que celles-ci ont valeur de test, ce qui va l'amener à dissimuler le matériel pathologique qui aurait tout à gagner à être extériorisé, comme dans le cas que nous avons cité plus haut. On pourrait objecter que faire sortir un patient pour lequel le traitement institutionnel semble loin du terme risque dans bien des cas de nous amener à négliger la dangerosité potentielle. En fait, si cette démarche est le fruit d'une réflexion collective et est intégrée dans un cheminement où se retrouvent le patient et le thérapeute, le risque semble mince car l'entrée en situation de violence correspond souvent à une élaboration que bien des indices peuvent nous révéler si le contact et le lien transférentiel est réel. C'est d'ailleurs seulement la reconnaissance de l'authenticité de ce lien qui peut nous permettre de tenter de telles démarches.

\section{BIBLIOGRAPHIE}

CORNFIELD. R.B. et FIELDING, S.D. (1980) : "Impact of the Threatening Patient on ward communications ", AM. J. Psychiat., 137:5, mai 1980.

DUBOR, P. et BERGERET, J. (1979) Psychologie pathologique. Masson. Paris, 1979, p. 165-185.

ESCANDE, M. et GOLDBERGER, E. : “Compréhension des psychoses de l'adulte dans une perspective Kleinienne, " évol. psychiat..

GOFFMAN, E. (1961) : Asylums, Doudleday and Co., New York.

HAY, D. et OREN, D. (1972) : "The Psychological stresses of intensive care unit nursing ", Psychosom. Med. 34, 109-118.

HOGHMANN, J. (1971) : "Pour une psychiatrie communautaire, "Éditions du Seuil, Paris.

HOCHMANN, J. (1981) : "La peur du fou ". Santé mentale au Qué.., vol. VI, no.1, juin 1981 .

KOECHLIN, Ph. (1968): " Psychothérapie institutionnelle au regard du malade», Inf. psychia., vol. 44 no 1.

LION, J.R. et PASTERNAK, S.A. (1973) : "Counter Transference Reactions to Violent patients ». AM. J. Psychiat.. 130:2. février 1973.

LION, J.R. et COLL. (1976) : "A violence clinic : three years' experience ", AM. J. Psychiat, 133:4, april 1976.

RACAMIER, P.C. (1970) : "Le soin institutionnel des psychotiques : nature et fonction ", Inf. psychiat., vol. 46 no 8.

RACAMIER, P.C. (1974) : "Psychothérapie dans les psychoses ", EMC 37819, Alo.

RACAMIER, P.C. (1976) : "L'interprétation psychiatrique des scizophrénies ", EMC 37291, A10.

RHINE, MN, MAYERSON, P., (1971) : "Crisis hospitalization within a psychiatric emergency service ", AM. J. Psychiat., $127: 1386-1391$. 
ROSEN, H. (1975) : "The impact of the psychiatric intensive care unit on patients and staff ", AM. J. Psychiat., 132:5, mai 1975.

SEARLES (1975) : "L'effort pour rendre l'autre fou 》, Tr. Fr. B. Bost, Nouvelle Revue Fr. de psychologie, no 12 p. 23-49.

SEGAL, H. (1969) : « Introduction à l'œuvre de Mélanie Klein, P.U.F., Paris, 4e ed., 1980.

VIRKKUNEN, M. (1974) : "Observations on violence in schizophrenia », Acta. Psychiat. Scand., vol. 50, 144-161.

WEISMAN, G., FEIRSTEIN, A., THOMAS, C. (1969) : «Three day hospitalization, a model for intensive intervention ", Arch. Gen. Psychiat., 21, 620-629. 